\title{
JOM-4S Overhauser Magnetometer and Sensitivity Estimation
}

\author{
Xiaorong Gong, Shudong Chen and Shuang Zhang * \\ College of Electronic Science and Engineering, Jilin University, Changchun 130012, China; \\ gongxr21@mails.jlu.edu.cn (X.G.); chenshudong@jlu.edu.cn (S.C.) \\ * Correspondence: zhangshuang@jlu.edu.cn
}

\section{check for}

updates

Citation: Gong, X.; Chen, S.; Zhang, S. JOM-4S Overhauser Magnetometer and Sensitivity Estimation. Sensors 2021, 21, 7698. https://doi.org/10.3390/s21227698

Academic Editor: Fabio Viola

Received: 27 October 2021

Accepted: 17 November 2021

Published: 19 November 2021

Publisher's Note: MDPI stays neutral with regard to jurisdictional claims in published maps and institutional affiliations.

Copyright: (c) 2021 by the authors. Licensee MDPI, Basel, Switzerland. This article is an open access article distributed under the terms and conditions of the Creative Commons Attribution (CC BY) license (https:// creativecommons.org/licenses/by/ $4.0 /)$.

\begin{abstract}
The Overhauser magnetometer is a scalar quantum magnetometer based on the dynamic nuclear polarization (DNP) effect in the Earth's magnetic field. Sensitivity is a key technical specification reflecting the ability of instruments to sense small variations of the Earth's magnetic field and is closely related to the signal-to-noise ratio (SNR) of the free induction decay (FID) signal. In this study, deuterated ${ }^{15} \mathrm{~N}$ TEMPONE radical is used in our sensor to obtain high DNP enhancement. The measured SNR of the FID signal is approximately $63 / 1$, and the transverse relaxation time $T_{2}$ is $2.68 \mathrm{~s}$. The direct measurement method with a single instrument and the synchronous measurement method with two instruments are discussed for sensitivity estimation in time and frequency domains under different electromagnetic interference (EMI) environments and different time periods. For the first time, the correlation coefficient of the magnetic field measured by the two instruments is used to judge the degree of the influence of the environmental noise on the sensitivity estimation. The sensitivity evaluation in the field environment is successfully realized without electrical and magnetic shields. The direct measurement method is susceptible to EMI and cannot work in general electromagnetic environments, except it is sufficiently quiet. The synchronous measurement method has an excellent ability to remove most natural and artificial EMIs and can be used under noisy environments. Direct and synchronous experimental results show that the estimated sensitivity of the JOM-4S magnetometer is approximately $0.01 \mathrm{nT}$ in time domain and approximately $0.01 \mathrm{nT} / \sqrt{\mathrm{Hz}}$ in frequency domain at a 3 s cycling time. This study provides a low-cost, simple, and effective sensitivity estimation method, which is especially suitable for developers and users to estimate the performance of the instrument.
\end{abstract}

Keywords: Overhauser magnetometer (OVM); sensitivity; proton magnetometer; dynamic nuclear polarization (DNP); scale quantum magnetometer

\section{Introduction}

The proton magnetometer is a scalar quantum magnetometer that is based on the Larmor precession of hydrogen protons in the Earth's magnetic field. In accordance with different polarization modes of hydrogen protons, proton magnetometers can be divided into two types: the first type is the classical proton precession magnetometer (PPM), which was first implemented by Packard, Varian, and Waters [1-3]. This type of magnetometer uses a bias magnetic field perpendicular to the Earth's magnetic field to orient the hydrogen proton moment to the direction of the combined magnetic field. After quickly cutting off the polarizing current and the bias magnetic field disappears, the proton magnetic moment will rotate around the Earth's magnetic field $B_{0}$ with an angular velocity $\omega=\gamma_{\mathrm{p}} B_{0}$, and $B_{0}$ can be obtained through an accurate calculation of $\omega[4,5]$. The second type is the Overhauser magnetometer (OVM) that is based on the Overhauser effect researched by Overhauser, Abragam, and Solomon [6-10]. It uses the resonance of electrons to transfer to the resonance of the hydrogen nucleus, thereby enhancing its magnetic moment. The subsequent process is consistent with the PPM. Obviously, the OVM is similar to the PPM, except that the sensor design and the polarization strategies are different. 
In the 1990's, France [11-13], Canada [14], and Russia [15,16] developed the OVM. In recent years, China has conducted a series of studies on the development of the OVM [17-22]. Compared with the PPM, the OVM has the following advantages. The OVM uses electrons to orient the nuclear magnetic moment. Thus, it has an extremely high polarization efficiency. The power consumption is less than $2 \mathrm{~W}$ at a $3 \mathrm{~s}$ cycling time, which is especially suitable for portable measurement. The dynamic nuclear polarization (DNP) effect enhances the free induction decay (FID) signal so that the signal-to-noise ratio (SNR) can reach approximately 100, making the OVM more sensitive than the PPM. The lateral relaxation time $T_{2}$ of the PPM is usually less than $1.0 \mathrm{~s}$, whereas that of the OVM can usually reach $2.0 \mathrm{~s}$ or more due to the different working materials of the sensors. This condition greatly improves the SNR of the late signal and further improves the sensitivity. On the basis of the above advantages, the OVM is widely used in total magnetic field measurements, such as space magnetic detection [11-13,23], magnetic anomaly detection [24], mineral prospecting [24], gas pipeline detection [25], geophysical exploration [26], archaeological detection [27], and geomagnetic observatory [14].

Same as the classical PPM and the optically pumped magnetometer [28-35], the technical specifications of the OVM include resolution, sensitivity, (absolute) accuracy, measurement range, gradient tolerance, cycling rate, drift, temperature range, and power consumption. In these specifications, sensitivity is a key technical specification of magnetometers for practical applications. Sensitivity reflects the relative uncertainty of instrument readings, and the fluctuation of the magnetometer reading is similar to Gaussian white noise. This condition is why sensitivity is often named noise level and can be characterized by standard deviation (STD) or power spectral density (PSD) [11-13]. Theoretically, when the STD is greater than the change in the measured micromagnetic anomaly, the measured micro magnetic anomaly will be submerged in the reading noise and cannot be detected.

Sensitivity is a characteristic of the magnetometer itself and should be measured in an undisturbed environment $[17,28-31]$. However, the environment without electromagnetic interference (EMI) is difficult to obtain. Therefore, investigating the sensitivity estimation method in an unshielded environment is necessary. This method is convenient for magnetometer users and developers to evaluate the performance of the instrument [32-35].

The sensitivity of the OVM is approximately an order of magnitude higher than that of the PPM, which can usually reach $0.015 \mathrm{nT} / \sqrt{\mathrm{Hz}}$ at $1 \mathrm{~Hz}$ [24]. Determining the sensitivity of homemade and commercial instruments through simple comparisons is difficult due to its high sensitivity [19]. Choosing a relatively stable piece of data to estimate the sensitivity to some extent eliminates the influence of diurnal variation on the sensitivity estimation, but the residual low-frequency components and high-frequency interference components still affect the sensitivity estimation [18]. When instruments with different sensitivities are not synchronized, the enhanced noise caused by subtracting data with each other degrades the accuracy of sensitivity estimation [22]. The fourth-order difference method can well eliminate the interference of low-frequency components, such as diurnal variation, but still cannot eliminate the high-frequency component interference [36]. The zero magnetic space is usually used for sensitivity estimation. However, it is difficult to obtain for ordinary users and developers, and the device that generates the magnetic field brings noise, which deteriorates the sensitivity estimation $[18,19]$. In summary, the estimated sensitivity is often inaccurate because no systematic, scientific, and effective evaluation method is available. The correlation coefficient of measurement results is introduced to quantitatively evaluate the effect of EMI noise on the reading noise of the magnetometer and to solve these problems. The direct measurement method with a single instrument and the synchronous measurement method with two instruments are discussed for sensitivity estimation in time and frequency domains under different EMI environments and different time periods.

The JOM-4S OVM is a portable magnetometer developed by the College of Electronic Science and Engineering of Jilin University over a decade. This study first introduces the JOM-4S OVM and describes it in detail, including its sensor and electronics. The application conditions of the direct method and synchronous method are discussed by introducing 
the correlation coefficient. The sensitivity under noisy and quiet environments by using two estimation methods at time and frequency domains is estimated.

\section{Physical Principles}

The core of the OVM sensor is the working material containing electrons and hydrogen protons. The working principle of the OVM sensor can be summarized into three stages: (1) excited by the radio frequency (RF) electromagnetic field, the electrons in free radicals produce electron spin resonance (ESR); (2) electron resonance drives hydrogen protons in solvent to produce nuclear magnetic resonance (NMR); (3) Larmor precession of hydrogen nuclei in solvent.

\subsection{ESR}

When deuterated ${ }^{15} \mathrm{~N}$ nitrogen oxygen radical TEMPONE dwells in an ambient $B_{0}$ magnetic field, which is in the oz direction, the Hamiltonian of the electron spin $S$ and the ${ }^{15} \mathrm{~N}$ nuclear spin $\mathrm{K}$ can be described as [37]

$$
\mathrm{H}=-\gamma_{\mathrm{S}} B_{0} S_{z}-\gamma_{\mathrm{K}} B_{0} \boldsymbol{K}_{z}+A \boldsymbol{S} \cdot \boldsymbol{K}
$$

where $A$ is a hyperfine coupling constant of the two-spin system. $\gamma_{\mathrm{S}}$ and $\gamma_{\mathrm{K}}$ are the gyromagnetic ratios of the electron and ${ }^{15} \mathrm{~N}$ nuclear, respectively. For the ${ }^{15} \mathrm{~N}$ TEMPONE nitroxide radical, $S=1 / 2$, and $K=1 / 2$. In the local field of ${ }^{15} N$, the unpaired electrons in the free radical are hyperfine coupled with a ${ }^{15} \mathrm{~N}$ nucleus, and Zeeman splitting occurs in the ambient Earth's field. In accordance with the Breit Rabi relation [38], in the presence of an external magnetic field, the energy levels of ${ }^{15} \mathrm{~N}$ radicals will split into four, and the energy level diagram is shown in Figure 1. The $\sigma$ transition (blue arrow) and the $\pi$ transition (red arrow) are excited by excitation in the same direction because the external magnetic field and excitation are perpendicular to the external magnetic field [37].

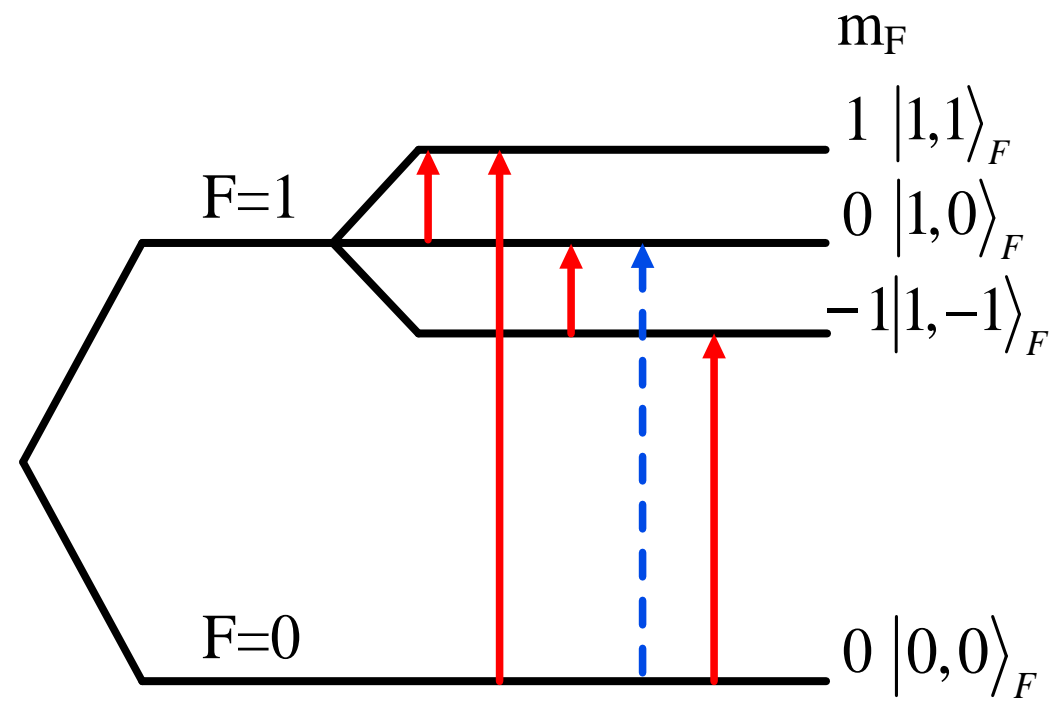

Figure 1. Energy levels of ${ }^{15} \mathrm{~N}$ nitroxide radical.

\subsection{Solomon's Equation}

In low fields, an effective means to amplify the NMR signal is through DNP by paramagnetic impurities. The amplification is obtained through the magnetic coupling between the protons and the unpaired electrons of the radicals in the OVM sensor. The coupled system has four energy levels, as shown in Figure 2 [8]. 


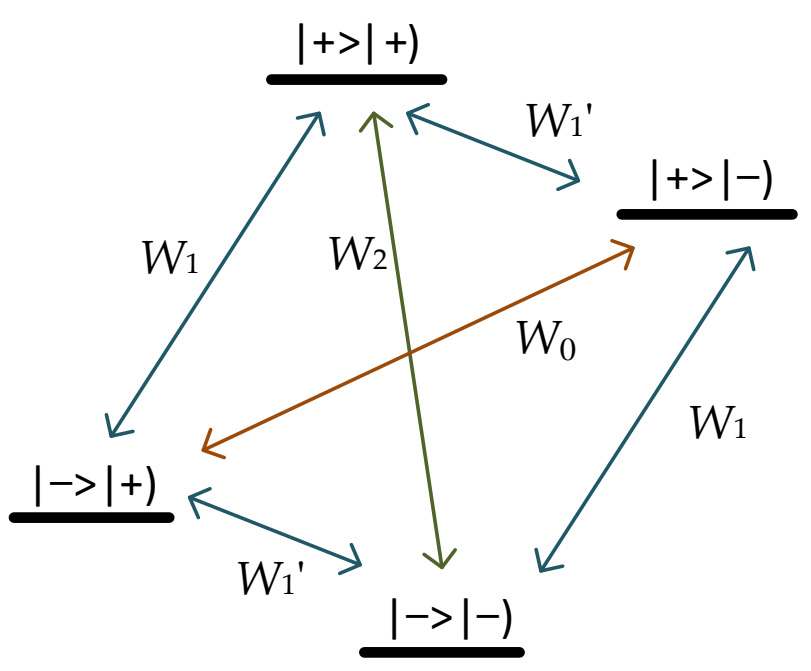

Figure 2. Energy diagram appropriate for coupled proton and electron, where $|+>|+),|->|-$ ), $|+>|-)$, and $|->|+$ ) are the four energy levels, respectively.

The first sign refers to electrons, and the second to protons, where $W_{0}, W_{2}, W_{1}{ }^{\prime}$, and $W_{1}$ are the transient probabilities per unit time between the four states. $W_{1}{ }^{\prime}$ denotes electronic transition, which realizes the transition of the electron from the eigenstate $\mid->$ to $|+\rangle$ (or $|+\rangle$ to $\mid->)$, and the process is completed by RF excitation. In the thermal equilibrium state, this transition will soon be saturated, but the two relaxation processes $W_{0}$ and $W_{2}$ ensure that the electron transitions from the eigenstate, $|+\rangle$ to $\mid->$ (or $\mid->$ to $|+\rangle$ ). The electronic transition $W_{1}{ }^{\prime}$ drives the proton $W_{1}{ }^{\prime}$ to transition, realizing the transition of the proton from the eigenstate $I-)$ to $I+)($ or $I+)$ to $I-)$ ). The two relaxation processes $W_{0}$ and $W_{2}$ also ensure that the proton goes from the eigenstate $I+$ ) transition to $I-$ ) (or $I-$ ) to $I+)$ ), avoiding the saturation of the proton transition. The proton polarization is increased, and the direction is toward the geomagnetic field. The overall DNP enhancement of $E$ is defined as [39]

$$
E=\frac{\left\langle I_{Z}\right\rangle}{I_{0}}=1-\xi \cdot s \cdot f \frac{\left|\gamma_{s}\right|}{\gamma_{n}}
$$

where $\left\langle I_{z}\right\rangle$ is the expectation value of the DNP, and $I_{0}$ is its thermal equilibrium value. $\gamma_{n}$ and $\gamma_{s}$ are the gyromagnetic ratios of the nuclei and electron, respectively. $\xi$, $f$, and $s$ are the coupling factor, leakage factor, and saturation factor, respectively. In the case of a pure scalar hyperfine dipolar coupling between the electronic spin $s$ and the nuclear spin $I$, $\xi=-1$. The value of $f$ determines the contribution of electrons to nuclear relaxation. In an ideal case, $f=1$, the nuclei relaxation is completely controlled by radical electrons at this time. The saturation factor $s$ is a measure of the saturation of the electronic transition. Ideally, the value of $s$ is 1 when the electronic transition is fully saturated. Thus, in the ultralow field, the value that can be obtained by DNP is determined by $\gamma_{s} / \gamma_{k}$, and the theoretical DNF factor is 660 [39]. In low fields, such as the Earth's magnetic field, several thousand times higher than its thermodynamic equilibrium can be obtained [14-27,36-40].

\subsection{Lamor Precession}

When the electron resonance transition is saturated, the resonant proton magnetic moment $M_{0}$ will precess to the direction of the total magnetic field $B\left(B_{0}+B_{p}\right)$. Then RF polarization $B_{\mathrm{RF}}$ and DC polarization $B_{p}$ are removed, and the excited protons will precess around the Earth's field $B_{0}$, named the Larmor precession. During Larmor precession, the relation between the angular frequency of Larmor precession $\omega_{0}$ and the Earth's field $B_{0}$ is [4]

$$
\omega_{0}=\gamma_{p} B_{0}
$$


where $\gamma_{p}=2.67515255 \times 10^{8} \mathrm{~T}^{-1} \mathrm{~s}^{-1}$ is the gyromagnetic ratio of the hydrogen nucleus. As long as the frequency value of the Larmor signal is measured with a frequency meter, the Earth's magnetic field can be accurately calculated.

\section{Construction of JOM-4S OVM}

\subsection{Brief Introduction of JOM-4S OVM}

As shown in Figure 3, the JOM-4S OVM consists of two parts, the instrument console and the sensor. The console includes four parts: analog board (Figure 4a), digital board (Figure 4b), key display board, and power board.

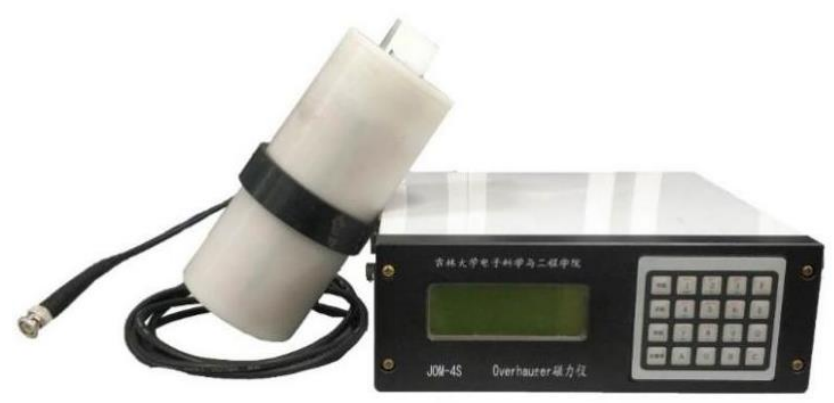

Figure 3. JOM-4S OVM.

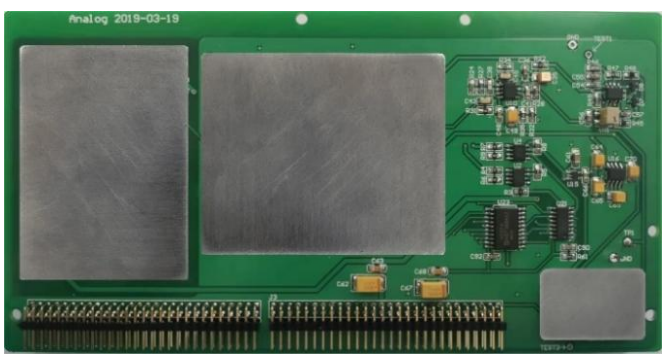

(a)

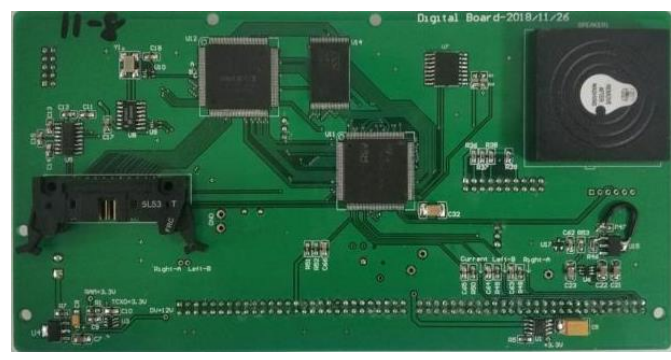

(b)

Figure 4. Analog board (a) and digital board (b) of JOM-4S OVM.

As shown in Figure 5, the Advanced RISC Machines (ARM) controls the Complex Programmable Logic Device (CPLD) to execute DC polarization, RF polarization, receiving, and tuning control. The sensor coil is shared by DC polarization and reception. The tuning capacitor and sensor coil form a LC parallel resonance to amplify the FID signal, and then the signal is sent to the last stage amplifier. The compared signal is sent to the CPLD to be counted. The count value is transmitted to the ARM to calculate the magnetic field. The rectified signal is sent to the A/D built-in ARM for signal quality evaluation.

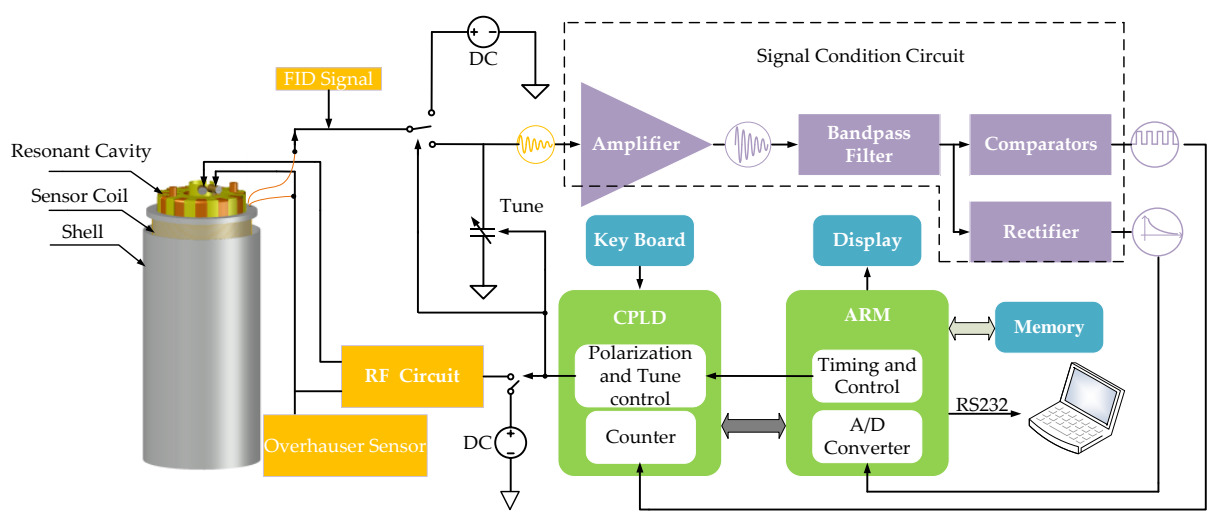

Figure 5. Block diagram of JOM-4S OVM. 


\subsection{Sensor Design}

The heart of the OVM is the sensor, which is mainly composed of two parts: an RF resonant cavity and a low-frequency coil. The coaxial resonant cavity is used to obtain a highly uniform transverse circular polarization RF magnetic field to resonate the unpaired electron in the cavity. The low-frequency coil is sleeved outside the resonant cavity to generate a bias field and receive the FID signal. The components of the OVM sensor are shown in Figure 6.

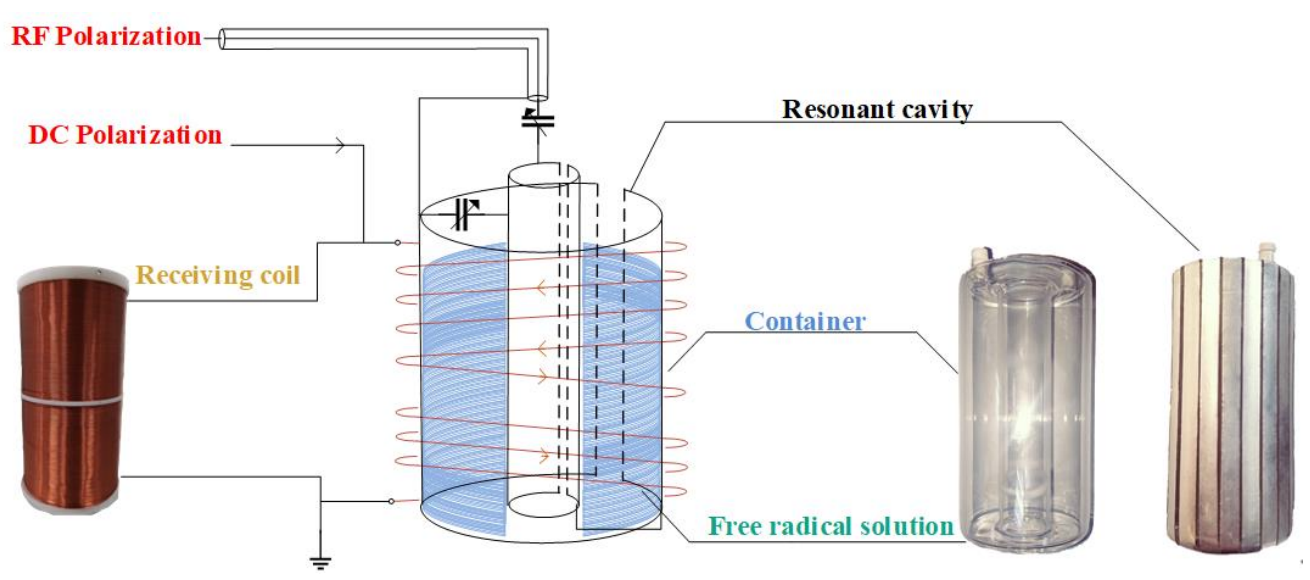

Figure 6. Components of the sensor.

Literature has shown that ${ }^{15} \mathrm{~N}-\mathrm{D}-4-\mathrm{Oxo}-2,2,6,6$-tetramethylpiperidine-1-oxyl $\left({ }^{15} \mathrm{~N}\right.$-D-oxo-TEMPO) has the highest enhancement factor compared with radicals such as ${ }^{14} \mathrm{~N}-\mathrm{H}$-oxo-TEMPO and ${ }^{14} \mathrm{~N}-\mathrm{H}$-TEMPOL [40]. So ${ }^{15} \mathrm{~N}$-D-oxo-TEMPO dissolved in solvent Diethylene glycol dimethyl ether (DME) is used in the JOM-4S Overhauser magnetometer for DNP enhancement. The molecular structure formula of ${ }^{15} \mathrm{~N}$-D-oxo-TEMPO is shown in Figure 7.

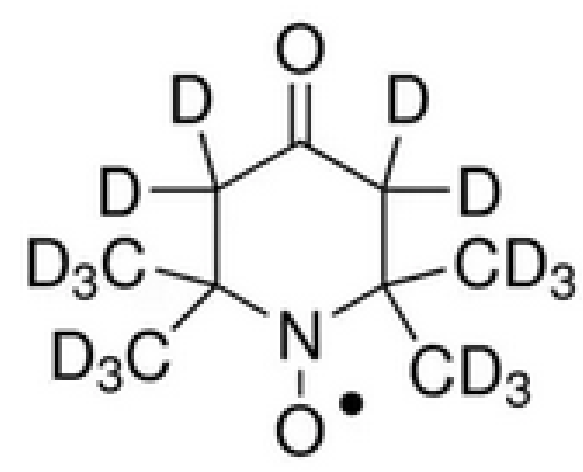

Figure 7. Molecular structure formula of ${ }^{15} \mathrm{~N}$-D-oxo-TEMPO.

The coaxial resonator is composed of a quartz bottle filled with a free radical solution and metal strips attached to the inside and outside of the container. Either the inner or outer conductor has gaps to prevent an eddy current. The length and the volume of the container are $112 \mathrm{~mm}$ and $175 \mathrm{~mL}$, respectively. A simulation model for the coaxial resonant cavity is established by using Ansoft HFSS to study the magnetic field distribution of the resonant cavity under the RF field, as shown in Figure 8a. The magnetic field distribution is shown in Figure $8 \mathrm{~b}$. The resonant cavity generates a uniform circularly polarized magnetic field. The S11 parameter of the resonant cavity is measured with an Agilent 8712ES network analyzer (Santa Clara, CA, USA). As shown in Figure 8c, after carefully adjusting the matching network, the $S 11$ parameter of the resonator is approximately $-45 \mathrm{~dB}$ measured 
at $58.8 \mathrm{MHz}$; its $-10 \mathrm{~dB}$ bandwidth covers approximately $58.823 \mathrm{MHz}$ to $58.777 \mathrm{MHz}$, and the $Q$ value is 1278 .

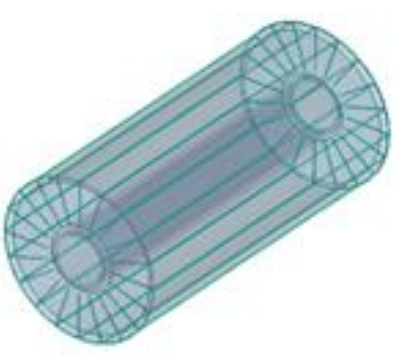

(a)

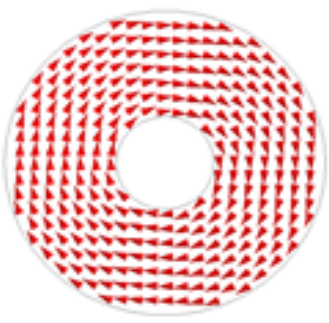

(b)

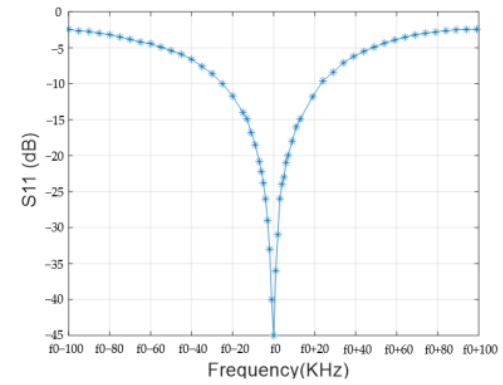

(c)

Figure 8. Coaxial cavity HFSS model (a), cross sectional magnetic field distribution (b) and S11 of resonant cavity (c).

The low-frequency coil is used for DC polarization and FID signal reception. The lowfrequency coil adopts a differential double-coil structure composed of two inverted coils in series, and the distribution of the magnetic field in the coil after DC polarization is shown in Figure 9a. When EMI occurs in the external environment, the induced voltages generated by the two coils are the same in magnitude and opposite in phase due to the differential structure. These voltages can effectively remove external common mode interference.

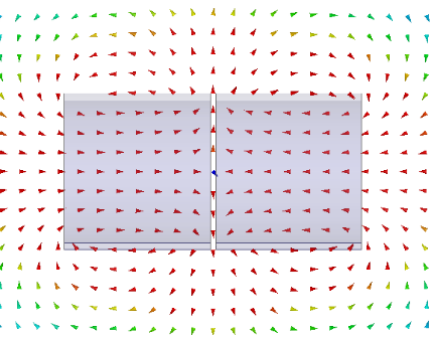

(a)

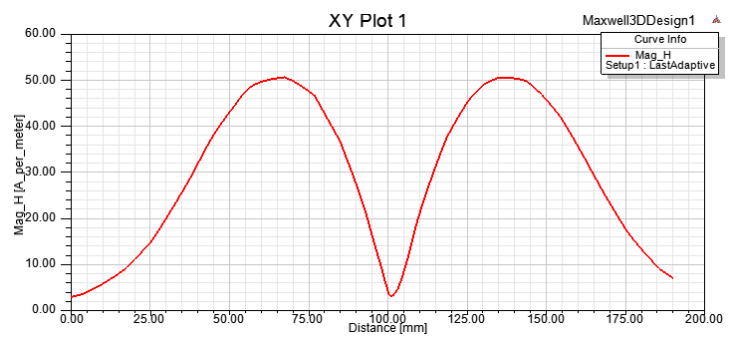

(b)

Figure 9. MAXWELL simulation of low-frequency coil (a) and axial magnetic field intensity distribution (b).

The design of the sensor geometry is mainly considered to be as small as possible for obtaining a high gradient tolerance; excessive coil turns will lead to extremely large inductance, which will reduce coil bandwidth and portability; the decrease in coil turns is accompanied by the decrease in signal; extremely thick wire diameter indicates extremely large volume and loss of portability; extremely small wire diameter leads to extremely large resistance, thereby increasing the thermal noise of the sensor. Considering the geometric size, gradient tolerance, coil bandwidth, signal amplitude, and sensor noise, the resultant number of turns is 700; the wire gauge is $0.53 \mathrm{~mm}$; the resistance is $22 \Omega$; the inductance is $35 \mathrm{mH}$; and the self resonant frequency is approximately $47 \mathrm{kHz}$ after shielding. As shown in Figure 9b, the maximum value of the DC polarization field is designed to be $50 \mu \mathrm{T}$.

\subsection{Analog Circuit Designs}

The main task of the analog circuit is signal conditioning to obtain a volt-level FID signal with a high SNR. As shown in Figure 10, the analog circuit is divided into seven functions: DC polarization, RF polarization, preamplifier, buffer amplifier, post amplifier, rectifier, and comparator. The square wave outputted by the comparator is inputted to the CPLD for counting, and the envelope signal obtained by the rectifier is used for signal quality evaluation.

A tuning capacitor connected in parallel with the sensor coil is needed to cover the measurement range of 20-120 uT and to achieve a resonance circuit, as shown in Figure 11. $r$ is the coil resistance, $L$ is the coil inductance, $C$ is the tuning capacitance, and $R_{d}$ is the matching resistance to limit the $Q$ value for avoiding self-excited oscillation. LC-parallel 
resonance is an important method for improving the SNR of the FID signal. The $Q$ values with and without $R_{d}$ are written in Equations (4) and (5).

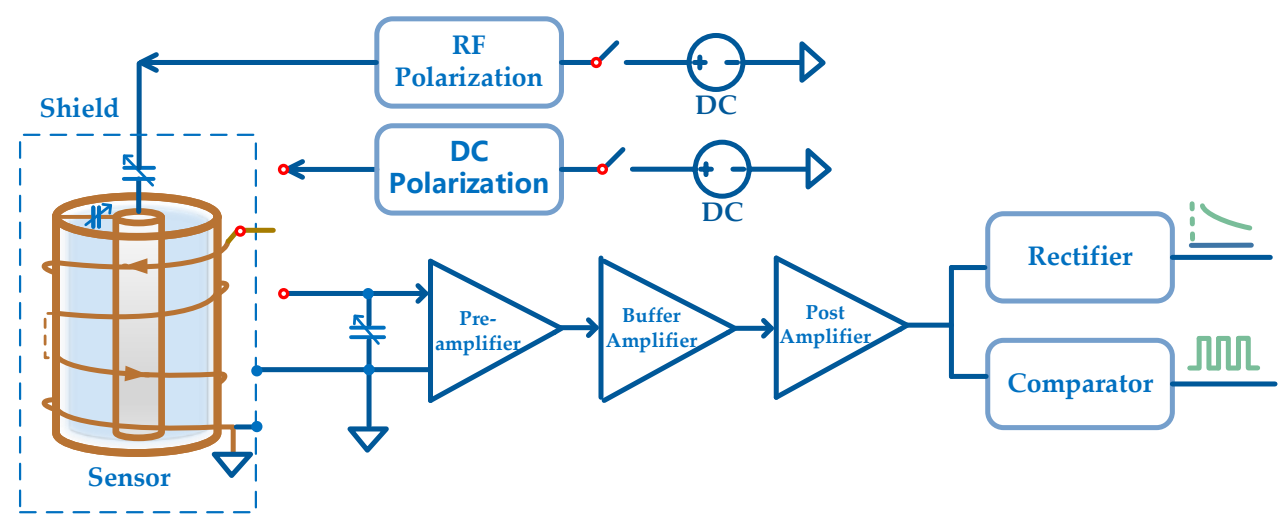

Figure 10. Block diagram of analog circuit.

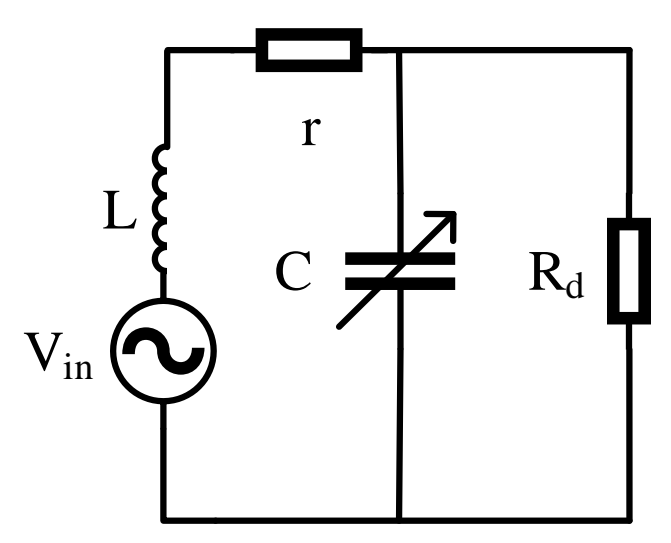

Figure 11. Equivalent circuit of sensor coil.

$$
\begin{gathered}
Q^{\prime}=\frac{R_{d}}{Q^{2} r+R_{d}} Q \\
Q=\frac{L \omega}{r}
\end{gathered}
$$

The calculated $Q$ value of the sensor coil without $R_{d}$ in parallel is 22. After connecting $R_{d}$ in parallel, the $Q^{\prime}$ value is reduced to 20 .

The preamplifier is based on a low-noise amplifier with a $1.4 \mathrm{nV} / \sqrt{\mathrm{Hz}}$ voltage noise and a $0.1 \mathrm{pA} / \sqrt{\mathrm{Hz}}$ current noise at $1 \mathrm{kHz}$. High accuracy and temperature stability resistors are used throughout the amplifier chain to reduce the addition of Johnson noise. The last stage-amplified signal is sent to the comparator and rectifier and is then sent to the ARM for counting and signal quality evaluation. The test scheme of the indoor comparator circuit and rectifier circuit is shown in Figure 12. The $500 \mathrm{mV}, 2.3 \mathrm{kHz}$ frequency sinusoidal signal generated by the AFG-2225 Arbitrary Function Generator (Suzhou, China) is divided into $23 \mathrm{uV}$ by $1.2 \mathrm{M} \Omega$ and $56 \Omega$ resistors. The signals are measured with a DSOX3504T Digital Storage Oscilloscope (Santa Clara, CA, USA). As shown in Figure 13a, the TEST 1 output signal (yellow) with $3.5 \mathrm{~V}$ peak-to-peak values is shaped and rectified into a square signal (blue) and an envelope signal (green). Considering that the electromagnetic induction signal is proportional to the frequency and $Q$ value of the sensor coil, a second-order filter circuit is used to filter out high-frequency noise and flat the transmission characteristics of the sensor and amplifier chain. 


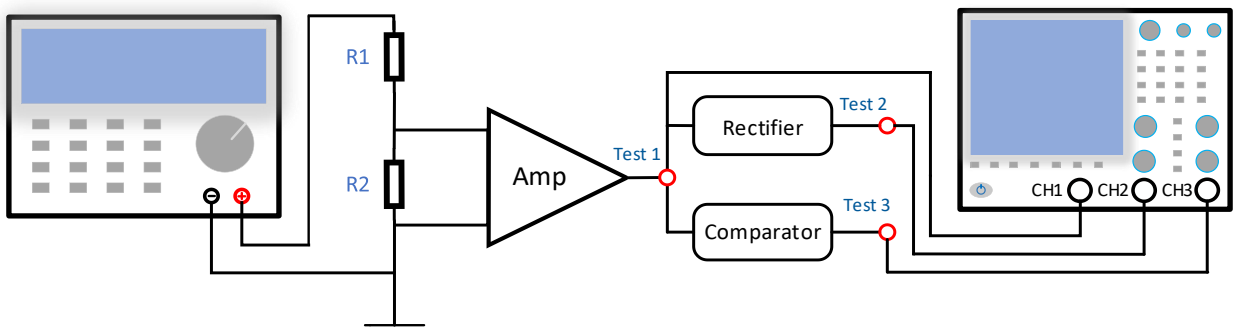

Figure 12. Schematic of envelope signal and shaping signal test.

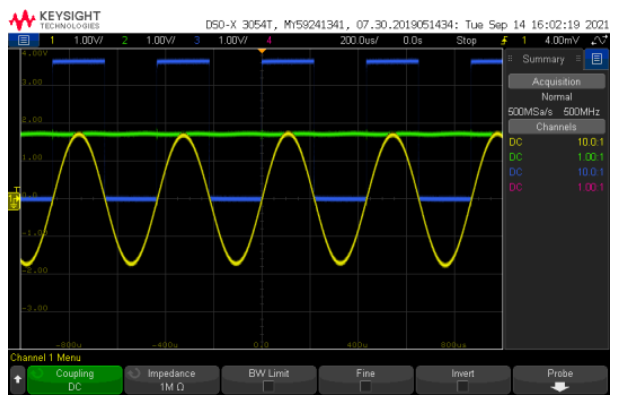

(a)

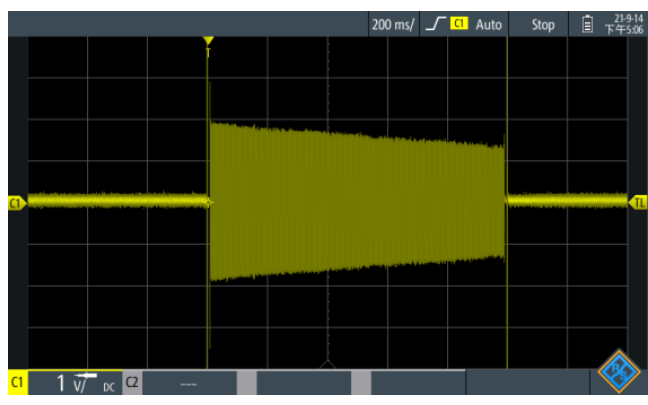

(b)

Figure 13. (a) Simulated FID signal, shaped signal, and envelope, (b) Measured FID signal.

The FID signal is measured by a digital oscilloscope Rohde \& Schwarz model RTH1002 (Munich, Germany) in the field environment shown in Figure 13b. The initial amplitude is $1.9 \mathrm{~V}$, and the attenuation constant is $2.68 \mathrm{~s}$. The STD of the measured noise is $30 \mathrm{mV}$, and the SNR is 1900/30 =63, which is twice larger than that of the PPM.

\subsection{Digital Circuit Designs}

As shown in Figure 14, the low-power microcontroller STM32 is used as MCU in the digital part to realize measurement control, data transmission, magnetic field calculation, storage, and other functions by controlling the CPLD, LCD, key, Flash, RAM, GPS, RS232, and other peripheral devices. The FLASH storage space is $256 \mathrm{M}$-bit, which can store up to $0.5 \mathrm{M}$ magnetic field values. The magnetometer can transmit measurement data to PC via RS232. RAM stores various initial setting states, such as the measurement mode. Key uses a $4 \times 5=20$ keyboard, LCD ZX1926M1A (Beijing, China) uses a $192 \times 64$ lowtemperature liquid crystal with heating function, and the GPS error is $2.5 \mathrm{~m}$. The CPLD mainly completes the measurement sequence control and counting.

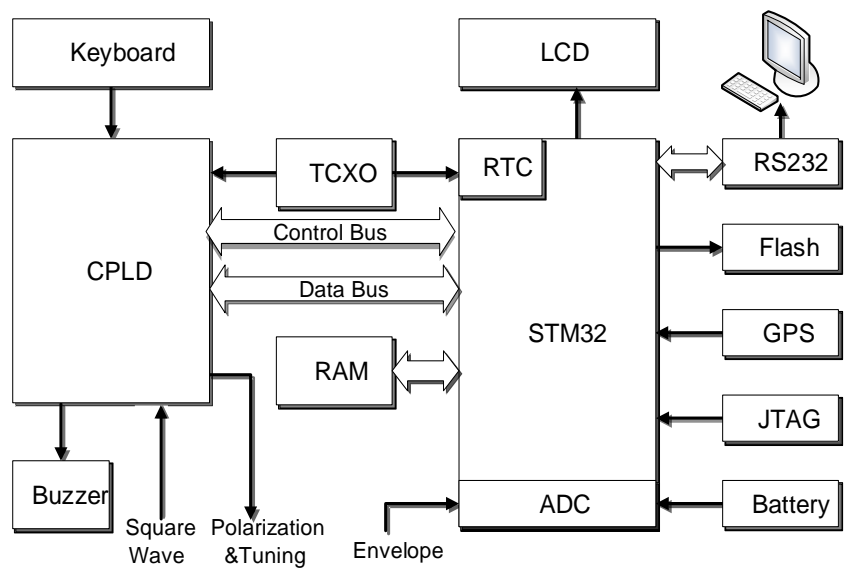

Figure 14. Digital circuit block diagram. 
JOM-4S is a slow-reading OVM. Its cycle time can be set from $3 \mathrm{~s}$ to $3600 \mathrm{~s}$. As shown in Figure 15, the polarization time and reception time are set to 2 and $1 \mathrm{~s}$ for all cycle times, respectively. DC and RF polarizations are performed at the same time.

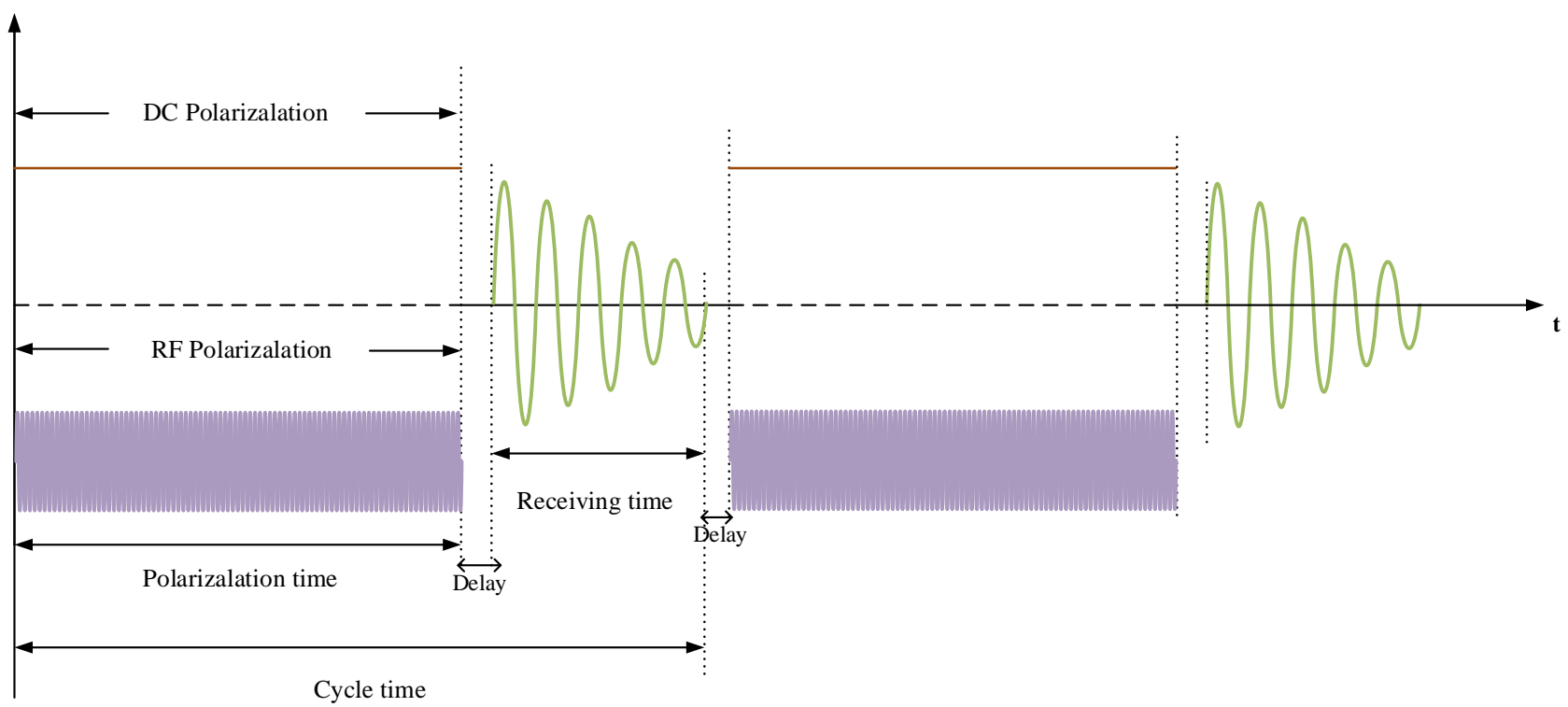

Figure 15. Sequence of polarization and reception controlled by ARM and CPLD.

As shown in Figure 16, $T$ is the preset gate time, which is controlled by signal quality. $T_{\mathrm{R}}$ is the actual measurement time and starts at the first rising edge in $T$ and ends at the last rising edge after $T . N$ is the number of cycles of the measured square wave signal, and $n$ is the number of cycles of the reference signal generated by a $4 \mathrm{MHz}$ temperaturecompensated crystal oscillator (TCXO) with $2 \mathrm{ppm}$ stability. $T_{\mathrm{R}}$ can be expressed by the following formula

$$
T_{\mathrm{R}}=N / f_{s}=n / f_{0},
$$

where $f_{s}$ is the frequency of FID signal. $f_{0}$ is the frequency of the reference signal. Thus, the frequency of the measured signal can be calculated by

$$
f_{s}=N f_{0} / n \text {. }
$$

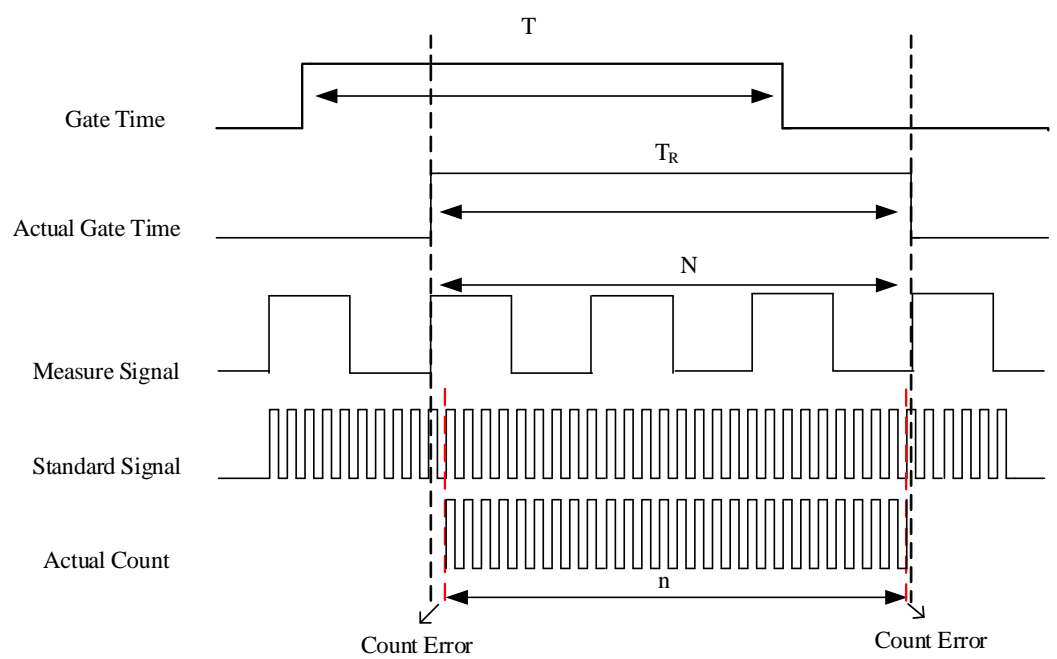

Figure 16. Schematic of frequency measurement.

The \pm 1 count error of $n$ can be ignored because $n$ much-greater-than $N$. 


\section{Sensitivity Estimation Methods}

As one of the important specifications of the OVM, sensitivity indicates the degree to which the measured value of the magnetometer deviates from the true value and can be calculated in terms of the STD of the reading noise of the instrument. In the environment with EMI, the measured total magnetic field $B(t)$ can be expressed as the addition of several components.

$$
B(t)=B_{0}+B_{l n}(t)+B_{h n}(t)+B_{n}(t),
$$

where $B_{0}$ is the DC component of the Earth's magnetic field, $B_{l n}$ is the low-frequency component consists of diurnal variation and drift, $B_{h n}$ is the high-frequency component consisting of environmental EMI, and $B_{n}$ is the reading noise of the OVM.

Theoretically, as long as $B_{l n}(t)=0$ and $B_{h n}(t)=0$ are satisfied, the sensitivity evaluation can be obtained by calculating the STD. However, this condition can only be satisfied in a zero magnetic environment. The following discussion focuses on the measurements in the field-unshielded environment where $B_{l n}(t)=0$ and $B_{h n}(t)=0$ are not satisfied. The direct measurement method and synchronization method are discussed to accurately evaluate the sensitivity in the time domain and spectral domain, respectively. Correlation analysis is used to evaluate the effect of the electromagnetic environment on sensitivity evaluation.

\subsection{Fourth-Order Difference}

When a single instrument is used to measure the Earth's field in a field environment, low-frequency components, such as diurnal variation and other low-frequency interferences, are inevitably found in the measured total field. Low-frequency components can be filtered out to prevent the effect on sensitivity estimation because the fourth-order difference function has high-pass filtering characteristics. The fourth-order difference function can be expressed as

$$
T_{i}=B_{i-2}-4 B_{i-1}+6 B_{i}-4 B_{i+1}+B_{i+2},
$$

where $T_{i}$ is the fourth-order difference of the measured magnetic field $B_{i}$.

After using the fourth-order difference method, we have $B_{l n}(t) \approx 0$. If high-frequency noise $B_{h n}(t) \approx 0$ can be satisfied, then sensitivity can be estimated accurately. However, $B_{h n}(t)$ existing in $B(t)$ cannot be eliminated because its characteristic is similar to reading noise $B_{n}(t)$. Whether the environment meets the requirement $B_{h n}(t) \approx 0$ will be discussed in Section 4.2.

\subsection{Correlation Analysis}

After the fourth-order difference of the magnetic field, only high-frequency EMI noise $B_{h n}$ and the reading noise of the instrument $B_{n}$ are included in $B(t)$. Theoretically, the high-frequency components of EMI noise $B_{h n}$ are the same in the magnetic field measured by the two synchronous instruments. We have

$$
T_{1}=T_{h n}+T_{n 1}, T_{2}=T_{h n}+T_{n 2},
$$

where 1 and 2 are the serial numbers of two instruments. $T_{1}$ and $T_{2}$ are the fourth-order differences of the magnetic field measured by two instruments. $T_{h n}$ is the fourth-order difference of the high-frequency components of EMI noise, and $T_{n 1}$ and $T_{n 2}$ are the reading noises of the two instruments. $T_{h n}, T_{n 1}$, and $T_{n 2}$ are independent of each other, and the statistical characteristics of $T_{n 1}$ and $T_{n 2}$ are the same. Therefore, the correlation coefficients $\rho$ of $T_{1}$ and $T_{2}$ can be expressed as

$$
\rho=\frac{\operatorname{Cov}(T 1, T 2)}{\sqrt{D(T 1) D(T 2)}}=\frac{D\left(T_{h n}\right)}{D\left(T_{h n}\right)+D\left(T_{n 1}\right)},
$$

where $\operatorname{Cov}\left(T_{1}, T_{2}\right)$ is the covariance of $T_{1}$ and $T_{2}$, and $D\left(T_{1}\right), D\left(T_{2}\right), D\left(T_{h n}\right)$, and $D\left(T_{n 1}\right)$ are the variances of $T_{1}, T_{2}, T_{h n}$, and $T_{n 1}$, respectively. As shown in Equation (11), the correlation coefficients of $T_{1}$ and $T_{2}$ depend on the variance of the EMI noise $T_{h n}$ and the 
reading noise $T_{n 1}$ of the instrument. When the EMI noise $T_{h n}$ is greater than the $T_{n 1}$ of the instrument, the correlation coefficient is close to 1 . When the EMI noise $T_{h n}$ is less than the reading noise $T_{n}$ of the instrument, the correlation coefficient is close to 0 . Therefore, the correlation coefficient can be used as an indicator for judging whether the influence of the environmental noise level on the sensitivity estimation is negligible.

\subsection{Direct Measurement Method in Time Domain}

The direct measurement method estimates the instrument sensitivity by measuring the magnetic field through a single instrument. This method is usually used for testing and estimating the sensitivity value in the zero magnetic space [17]. However, the zero magnetic field space is difficult to obtain, and the field test is susceptible to the influence of the diurnal variation of the Earth's field and EMI. Only a piece of approximately constant data can be usually selected for estimation so that the diurnal variation and external EMI can be approximately ignored. We use Equation (9) to perform the fourth-order difference on the measured data to eliminate low-frequency interference. In accordance with Equations (10) and (11), the correlation of the magnetic fields measured by two instruments is used to evaluate the influence of the high-frequency components of EMI noise $B_{h n}$. When the reading noise of the instrument is dominant, coefficients $\rho$ much-smaller-than 1 , we have $B_{h n}(t)$ much-smaller-than $B_{n}(t)$ (or $B_{h n}(t) \approx 0$ ). In the time domain, STD is used to indicate sensitivity, as shown in Equation (12).

$$
\sigma=\frac{1}{\sqrt{70}} \sqrt{\frac{1}{n-1} \sum_{i=1}^{n}\left(T_{i}-\bar{T}\right)^{2}},
$$

where $\bar{T}$ is the mean value of $T_{i}$, and $T_{i}$ is the fourth-order difference of $B_{i}$.

\subsection{Synchronization Method in Time Domain}

The synchronous measurement method refers to the simultaneous measurement of two instruments to obtain the sensitivity of one of the instruments. The synchronization method can remove the low-frequency components $B_{l n}(t)$ of the diurnal changes and effectively remove the high-frequency EMI $B_{h n}(t)$ during the subtraction process. Thus, we have $B_{l n}(t) \approx 0$ and $B_{h n}(t) \approx 0$. When the sensitivity of two sets is the same, the sensitivity of a single set can be expressed as Equation (13).

$$
\sigma=\frac{1}{\sqrt{2}} \frac{1}{\sqrt{70}} \sqrt{\frac{1}{n-1} \sum_{i=1}^{n}\left(T_{1 i}-T_{2 i}+\bar{T}_{2 i}-\bar{T}_{1 i}\right)^{2}},
$$

where $T_{1 i}$ and $T_{2 i}$ represent the fourth-order difference of magnetic field values measured by the two instruments in the synchronous mode, and $\bar{T}_{1 i}$ and $\bar{T}_{2 i}$ are the mean values of $T_{1 i}$ and $T_{2 i}$, respectively.

\subsection{PSD Estimation in Frequency Domain}

In Sections 4.3 and 4.4, the time domain sensitivity of the system is estimated by the STD of reading noise. In the frequency domain, the PSD of the magnetic field can be used to estimate the noise level of the instrument $[11,17]$. If the environment under test is sufficiently quiet, the fluctuation of the environmental magnetic field is lower than the noise of the magnetometer under testing. The spectral sensitivity can be calculated in accordance with Equation (14).

$$
P S D=|F F T(B(t)-\bar{B}(t))|,
$$

where $B(t)$ is the readings of the single magnetometer. 
If the environment under test is insufficiently quiet, difference readings of two synchronous instruments are used to eliminate the interference of electromagnetic environment. Spectral sensitivity can be calculated in accordance with Equation (15).

$$
P S D=\frac{1}{\sqrt{2}}\left|F F T\left(B_{1}(t)-B_{2}(t)-\bar{B}_{1}(t)+\bar{B}_{2}(t)\right)\right|,
$$

where $B_{1}(t)$ and $B_{2}(t)$ are the readings of the two synchronized magnetometers, respectively. Either in Equation (14) or Equation (15), the DC components of the measured magnetic field are all removed to reduce their influence on high-frequency components. In Equation (15), the Hanning-windowed Fourier transform is used to prevent spectrum energy leakage.

\section{Experimental Results and Discussions}

The experiments were conducted in a quiet environment and noisy environment and lasted for approximately $24 \mathrm{~h}$ to observe the diurnal variations of the Earth's magnetic field. The campus of Jilin University was chosen for the noisy environment experiment on 5 May 2021, and the rural area of the Jiutai District of Changchun City was chosen for the quiet environment experiment on 7 June 2021. The sensitivity estimation methods in the time and frequency domains were applied to evaluate the sensitivity of the JOM$4 \mathrm{~S}$ magnetometer.

\subsection{Sensitivity Estimation in Time Domain under a Quiet Environment}

Two synchronous JOM-4S magnetometers were used to observe geomagnetic diurnal variation in the rural area of Jiutai City at a cycle rate of $3 \mathrm{~s}$. The survey area was at the foot of Miaoxiangshan Mountain, far from the urban area, with a small EMI. Two instruments observed the value of the magnetic field for $24 \mathrm{~h}$. The fourth-order difference of the magnetic field and the difference value of the fourth-order difference are shown in Figure 17.

As shown in Figure 17a, the diurnal variation of the magnetic field reached the maximum at 3-5 p.m. and the minimum at 9-11 a.m. The whole diurnal variation fluctuated by approximately $50 \mathrm{nT}$. As shown in Figure 17b, the fourth-order difference of the two magnetic field values completely eliminates the diurnal variation. From 10:00 p.m. to 4:00 a.m., the peak value of the fourth-order difference of the magnetic field was less than $1.0 \mathrm{nT}$. In the rest of the time, the peak-to-peak value of the fourth-order difference of the magnetic field reached 5-6 nT. As shown in Figure 17c, the correlation coefficient of the fourth-order difference of S001 and S002 was less than 0.2 between 10:00 p.m. and 4:00 a.m., but near 1 at other times. This condition indicates that the reading noise $B_{n}(t)$ of the instrument dominated between 10:00 p.m. and 4:00 a.m. The sensitivity of the instrument was estimated to be 0.0090 and $0.0092 \mathrm{nT}$ with a direct measuring method between this period. In the rest of the time, the peak-to-peak value of the fourth-order difference of the magnetic field reached 5-6 nT, and the sensitivity estimated by the direct measuring method was 0.0417 and $0.0418 \mathrm{nT}$, which was greater than in the night. As shown in Figure 17d, two fourth-order differences were made to estimate the target sensitivity more accurately. The 24-h data and the 7-h data from 10:00 p.m. to 4:00 a.m. were applied with synchronization method to estimate the sensitivity as 0.0090 and $0.0086 \mathrm{nT}$, respectively.

The sensitivity of the magnetometer can be accurately estimated with the direct measuring method at night under a quiet environment as $0.009 \mathrm{nT}$ or with the synchronization method throughout $24 \mathrm{~h}$ as $0.009 \mathrm{nT}$. 


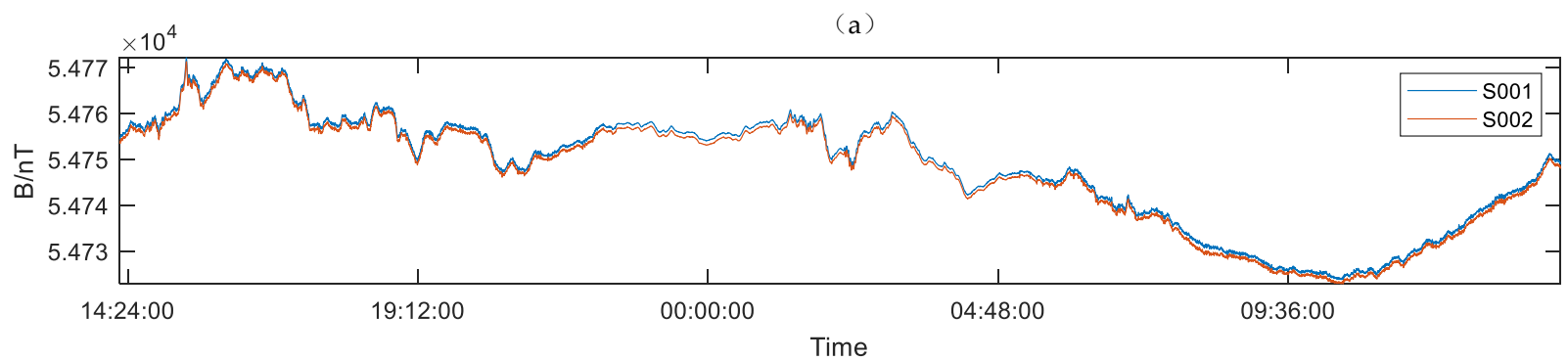

(b)

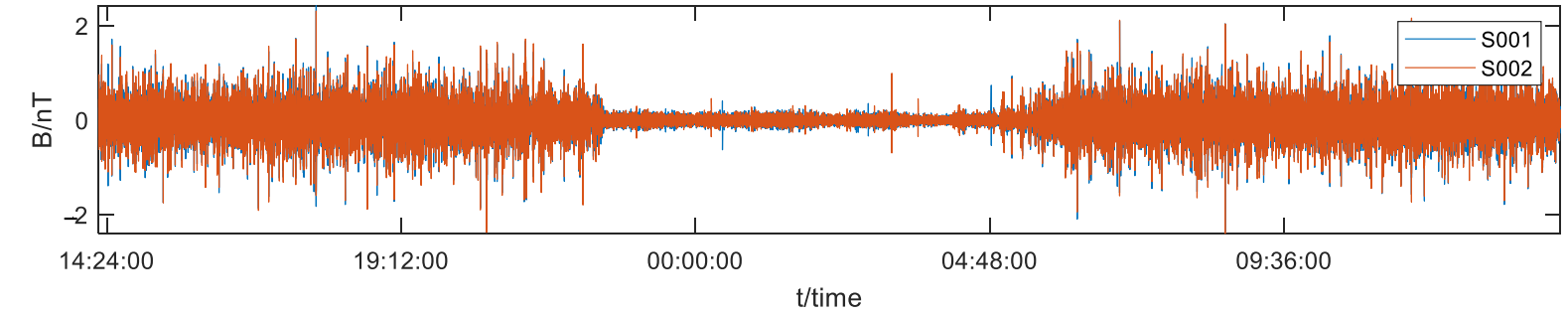

(c)

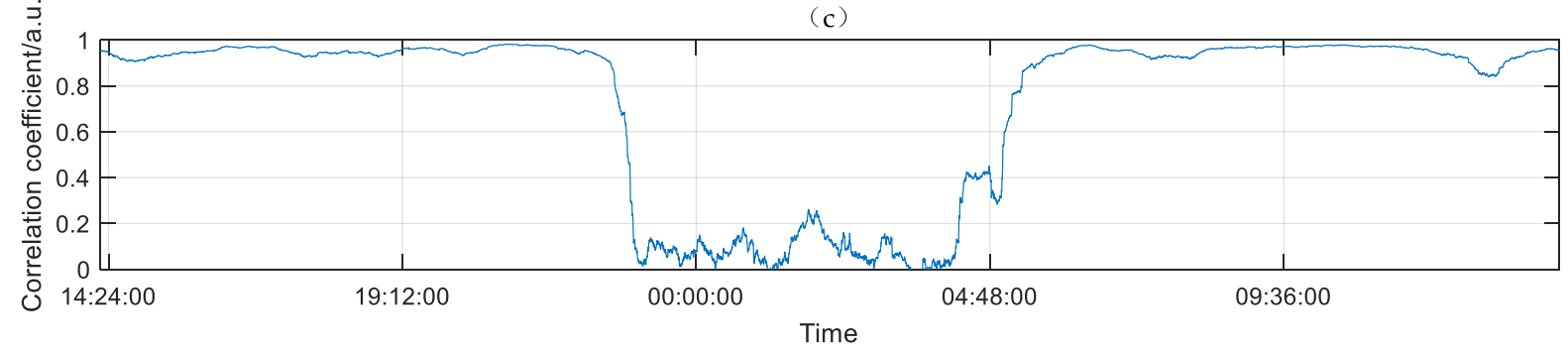

(d)

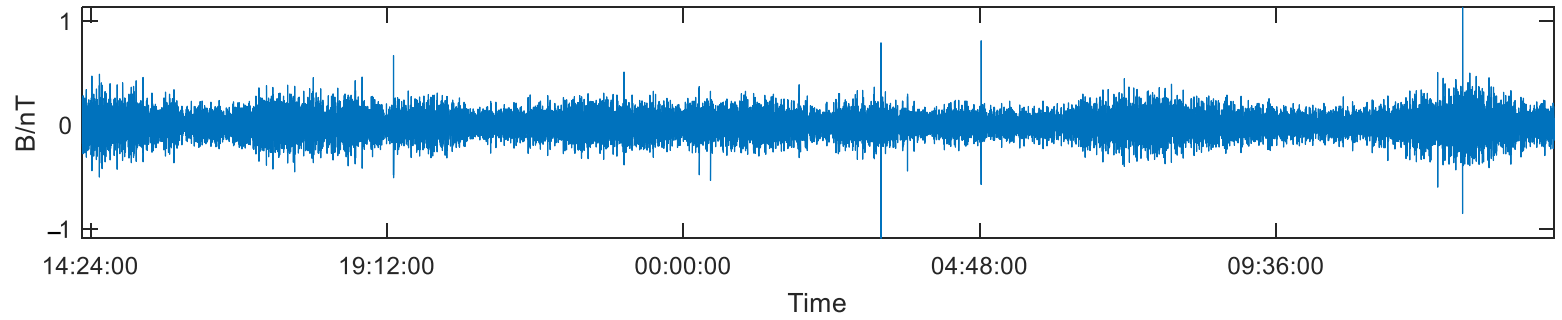

Figure 17. (a) $24 \mathrm{~h}$ magnetic field value, (b) fourth-order difference of the magnetic field, (c) correlation coefficient of S001 and S002, (d) difference value of the fourth-order difference.

\subsection{Sensitivity Estimation in Time Domain under a Noisy Environment}

Long-time measurement ( $24 \mathrm{~h}$ ) was conducted on the campus of Jilin University on 5 May 2021 at the same cycle rate of $3 \mathrm{~s}$ to analyze the influence of noise on the sensitivity evaluation of the magnetometer system. The campus environment is extremely complex, and the measurement results are affected by interference, cable interference, and automobile interference. Two instruments observed the value of the magnetic field for $24 \mathrm{~h}$. The fourthorder difference of the magnetic field and the difference value of the fourth-order difference are shown in Figure 18.

As shown in Figure 18, the magnetic field fluctuated up to $100 \mathrm{nT}$ in a short time during the day under high noise conditions. From 10:00 p.m. to 4:00 a.m., the fluctuation was extremely low. Therefore, the estimation of system sensitivity can only be conducted between 10:00 p.m. and 4:00 a.m. As shown in Figure 18b, the STDs of the fourth-order difference of the two magnetic field values reached 3.8684, 3.8677 nT in the daytime and $0.4239 \mathrm{nT}$ and 0.4217 nT from 10:00 p.m. to 4:00 a.m. Specifically, even at night, the fourthorder difference of the magnetic field value in the high-noise environment still reflected the environmental noise and cannot be used to estimate the sensitivity. As shown in Figure 18c, the correlation coefficient of the fourth-order difference of S001 and S002 was extremely near to 1 throughout the whole day. In other words, the EMI noise was greater than the 
reading noise of the instrument. Thus, the sensitivity of the instrument cannot be estimated by using the direct measurement method. As shown in Figure 18d, the synchronization method was chosen to estimate the sensitivity in accordance with Equation (13). Taking the data of $7 \mathrm{~h}$ from 10:00 p.m. to 4:00 a.m., the sensitivity of the system is estimated to be $0.0084 \mathrm{nT}$.
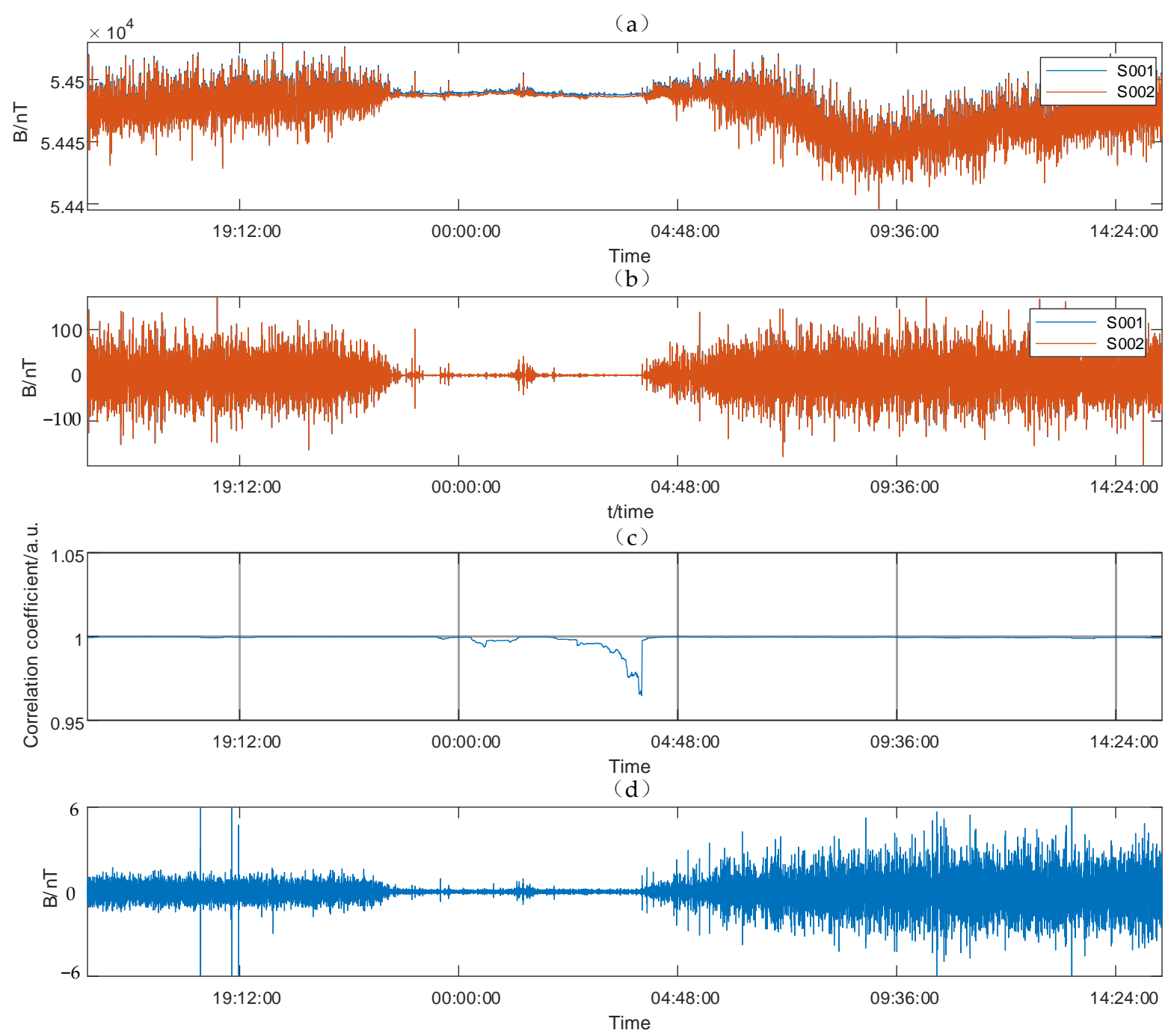

Figure 18. (a) $24 \mathrm{~h}$ magnetic field value, (b) fourth-order difference of the magnetic field, (c) correlation coefficient of fourth-order difference of S001 and S002, (d) difference value of the fourth-order difference.

\subsection{Sensitivity Estimation in Frequency Domain}

In accordance with the time domain analysis in Section 5.2, the EMI intensity was the lowest between 10:00 p.m. and 4:00 a.m. in a day. Thus, the magnetic field data in this time period are selected to estimate sensitivity. The direct spectrum estimation method of sensitivity was obtained by substituting the S001 or S002 time domain data in Equation (14) to calculate the PSD. The synchronous spectrum estimation method of sensitivity substitutes the time domain data difference of two instruments in Equation (15) to calculate the PSD.

As shown in Figure 19a, the PSDs of S001 and S002 in the low-frequency band were highly consistent. The form of PSD of S001-S002 was consistent with S001 and S002, but the amplitude was reduced by one order of magnitude due to the subtraction operation. These findings indicate that the diurnal variation and low-frequency interference are dominant in the low-frequency band and do not reflect the noise level of the instrument. In the 
high-frequency band between $0.3-0.5 \mathrm{~Hz}$, the PSD was enlarged, as shown in Figure 19b. The PSDs of S001, S002, and S001-S002 are independent of each other and tend to be $0.01 \mathrm{nT} / \sqrt{\mathrm{Hz}}$. This value is consistent with the case when the correlation coefficient is 0.2 in the time domain. This condition implies that the noise level of the instrument is dominant in the high-frequency band. The sensitivity estimation can be obtained by either the synchronous method or the direct method under a quiet environment, and $0.01 \mathrm{nT} / \sqrt{\mathrm{Hz}}$ is the frequency domain sensitivity of JOM-4S magnetometer.

(a)

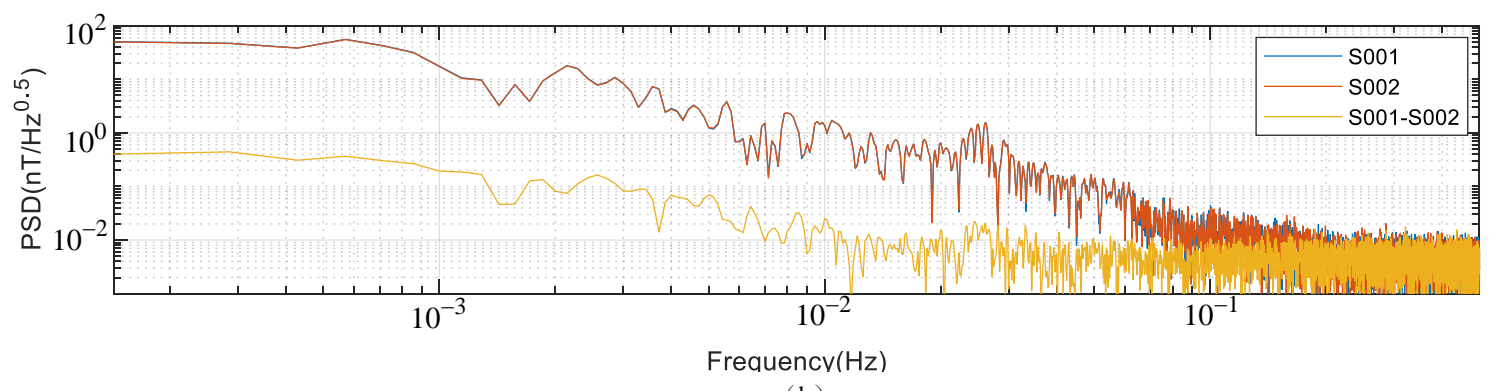

(b)

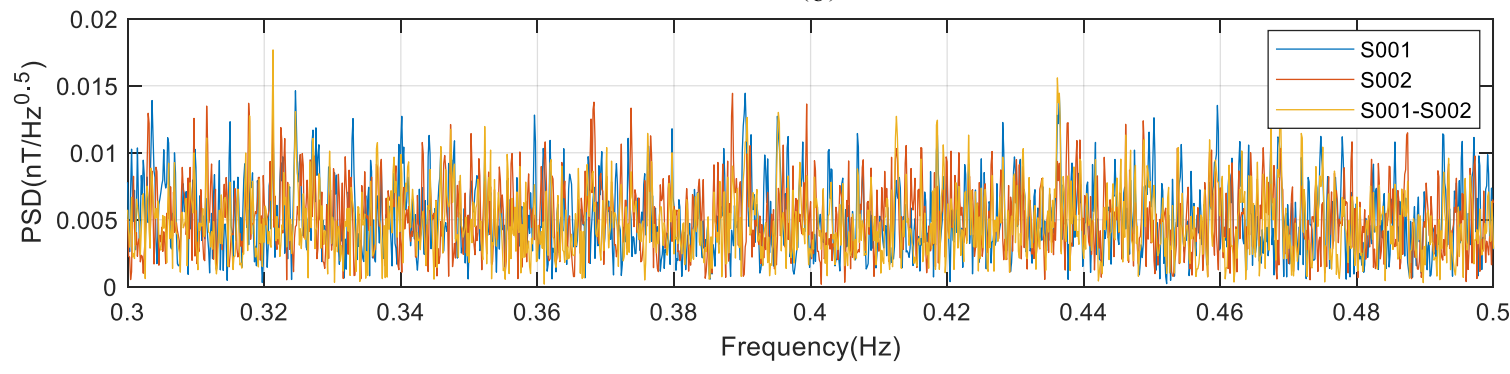

(c)

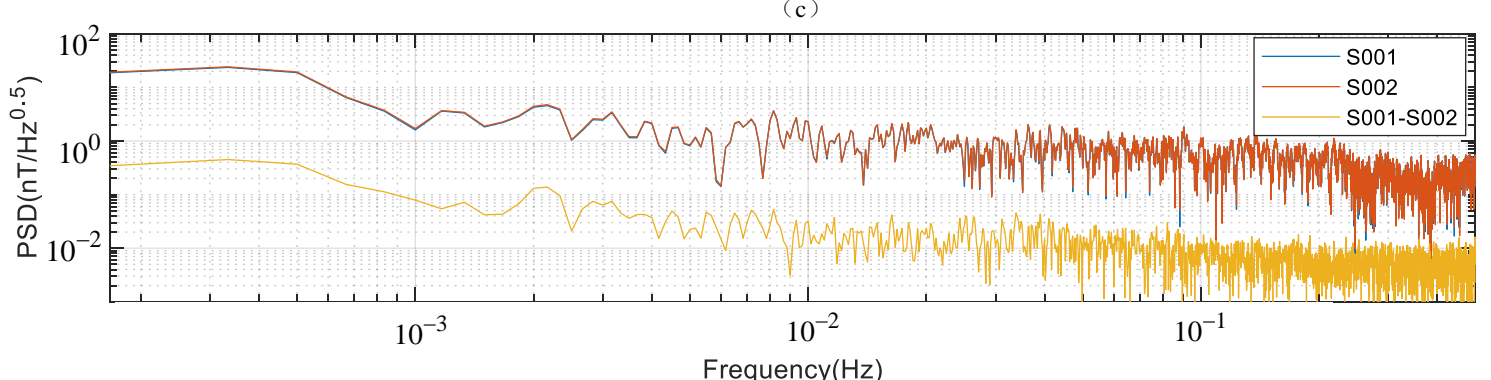

(d)

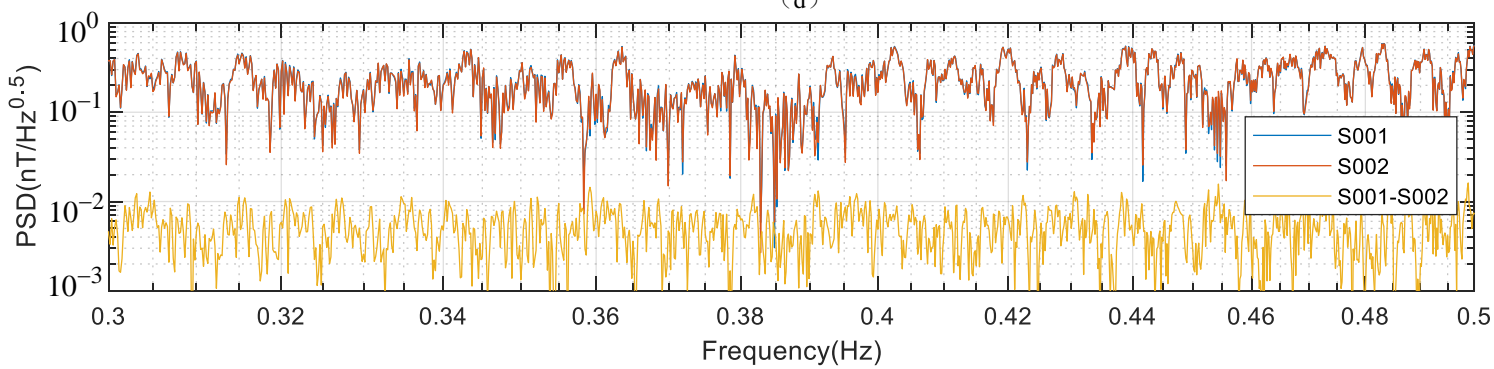

Figure 19. (a) PSDs under quiet environment, (b) high-frequency components of PSDs under quiet environment, (c) PSDs under noisy environment, (d) high-frequency components of PSDs under noisy environment.

As shown in Figure 19c,d, the same sensitivity evaluation experiments were implemented in the noisy environment. Different from the results in the quiet environment, S001 and S002 coincide in all frequency bands, indicating that the measured data of the two instruments are highly correlated. This condition is consistent with the case when the correlation coefficient is 0.999 in the time domain. Therefore, the direct estimation method fails in noisy environment, and the sensitivity evaluation can only be conducted through the synchronization method because the EMI noise is dominant at all frequency 
bands. The synchronization method can effectively eliminate the low-frequency and highfrequency interferences in the environment and obtain the same evaluation results as in the quiet environment.

\section{Conclusions}

The JOM-4S OVM, developed by the College of Electronic Science and Engineering of Jilin University, is briefly introduced, including its sensor and circuit. JOM-4S is a portable slow-reading OVM magnetometer, and its cycle time can be set from $3 \mathrm{~s}$ to $3600 \mathrm{~s}$. TEMPONE radical is used as an electron nuclear double-resonance working material in the proton-rich resonant cavity and significantly improves the DNP effect. The improved $Q$ value of resonant cavity can reach 1278 , resulting in the initial signal amplitude of $1.9 \mathrm{~V}$. The SNR of the FID signal can reach to 63/1 when the $1 \mathrm{~W}$ RF output power is polarized. The transverse relaxation time $T 2$ can reach $2.68 \mathrm{~s}$, greatly improving the average SNR of the FID signal in one cycle. In this study, the low-noise sensor, low-noise signal conditioning circuit, and equal-precision counting method jointly ensure that the JOM-4S magnetometer has an extremely high sensitivity.

The feasibility of the accurate sensitivity evaluation of the magnetometer in the field environment is discussed comprehensively through theoretical analysis and experimental verification. In the field environment, the nature of various noises that affect sensitivity evaluation and the mechanism of how these noises affect the accuracy of sensitivity estimation are investigated. A synchronization method using two instruments and the direct method using a single instrument can achieve accurate sensitivity estimation under the condition that the influence of environmental noise on the reading noise of the instrument is negligible. As a quantitative evaluation standard of the electromagnetic environment, the correlation coefficient of the reading noise of the two instruments is proposed. The analysis results show that the direct method cannot accurately estimate the sensitivity when the correlation coefficient approaches 1 but can accurately estimate the sensitivity when the correlation coefficient approaches 0 .

The sensitivity estimation experiments in the field environment show that the synchronization method can effectively eliminate most of the low-frequency and high-frequency EMIs in the environment. Thus, the synchronization method can achieve accurate sensitivity estimation as long as it is not in an extremely noisy environment. The experimental results show that only in an extremely quiet field environment can the correlation coefficient be close to 0 , and the sensitivity of the magnetometer can be accurately estimated by using the direct method. All experimental results agree well with the theoretical predictions. Thus, the sensitivity evaluation of the magnetometer can be evaluated in the field environment. This method is simple, effective, and low cost.

Sensitivity estimations in time and frequency domains are conducted to estimate the sensitivity in different EMI environments. The results show that the time domain sensitivity and the frequency domain sensitivity of the JOM-4S OVM are approximately $0.01 \mathrm{nT}$ and $0.01 \mathrm{nT} / \sqrt{\mathrm{Hz}}$ at a $3 \mathrm{~s}$ cycle rate, respectively. The sensitivity of the JOM-4S Overhauser magnetometer introduced in this article is comparable to that of the commercial magnetometer. Therefore, the JOM-4S OVM can measure the Earth's magnetic field with sufficient sensitivity and can be used in various fields of weak magnetic measurement.

Author Contributions: The authors confirm their contribution to the paper as follows: Conceptualization, X.G., S.C. and S.Z.; methodology, X.G., S.C. and S.Z.; software, X.G.; validation, X.G., S.C. and S.Z.; formal analysis, X.G., S.C. and S.Z.; investigation, X.G., S.C. and S.Z.; resources, X.G., S.C. and S.Z.; data curation, X.G., S.C. and S.Z.; writing-original draft preparation, X.G.; writing-review and editing, X.G., S.C. and S.Z.; visualization, X.G.; supervision, S.Z.; project administration, S.Z.; funding acquisition, S.Z. All authors have read and agreed to the published version of the manuscript.

Funding: This research was funded by the National Natural Science Foundation of China under grant number 61771218.

Institutional Review Board Statement: Not applicable. 
Informed Consent Statement: Not applicable.

Data Availability Statement: No new data were created or analyzed in this study. Data sharing is inapplicable to this article.

Acknowledgments: The authors would like to gratefully thank the anonymous reviewers for their time to review our article and their constructive comments. Any errors or omissions remain the sole responsibility of the authors.

Conflicts of Interest: The authors declare no conflict of interest.

\section{References}

1. Packard, M.E. Free nuclear induction in the earth's magnetic field. Phys. Rev. 1954, 93, 941.

2. Varian, R.H. Method and Means for Correlating Nuclear Properties of Atoms and Magnetic Fields. U.S. Patent US2561490A, 24 July 1951.

3. Waters, G.S. A measurement of the earth's magnetic field by nuclear induction. Nature 1955, 4484, 691. [CrossRef]

4. James, A.; Koehler, R. Proton precession magnetometers, revision 3. In Proceedings of the Industrial Control and Electronics Engineering (ICICEE), Comox, BC, Canada, 23-25 March 2012.

5. Mahavarkar, P.; Singh, S.; Labde, S.; Dongre, V.; Patil, A. The low cost Proton Precession Magnetometer developed at the Indian Institute of Geomagnetism. J. Instrum. 2017, 12, T05002. [CrossRef]

6. Overhauser, A.W. Polarization of nuclei in metals. Phys. Rev. 1953, 92, 411. [CrossRef]

7. Abragam, A. Overhauser effect in nonmetals. Phys. Rev. 1955, 98, 1729-1735. [CrossRef]

8. Solomon, I. Relaxation precesses in a system of two spins. Phys. Rev. 1955, 99, 559-565. [CrossRef]

9. Halse, M.E.; Callaghan, P.T. A dynamic nuclear polarization strategy for multi-dimensional Earth's field NMR spectroscopy. J. Magn. Reson. 2008, 195, 162-168. [CrossRef] [PubMed]

10. Guiberteau, T.; Grucker, D. EPR spectroscopy by dynamic nuclear polarization in low magnetic field. J. Magn. Reson. Ser. B 1996, 110, 47-54. [CrossRef]

11. Kernevez, N.; Glénat, H. Description of a high sensitivity CW scalar DNP-NMR magnetometer. IEEE Trans. Magn. 1991, 27, 5402-5404. [CrossRef]

12. Duret, D.; Bonzom, J.; Brochier, M.; Frances, M.; Leger, J.M.; Odru, R.; Salvi, C.; Thomas, T.; Perret, A. Overhauser magnetometer for the Danish Oersted satellite. IEEE Trans. Magn. 1995, 31, 3197-3199. [CrossRef]

13. Duret, D.; Leger, J.M. Performances of the OVH magnetometer for the Danish Oersted satellite. IEEE Trans. Magn. 1996, 32, 4935-4937. [CrossRef]

14. Hrvoic, I. Overhauser Magnetometers for Measurement of the Earth's Magnetic Field. In Proceedings of the International Workshop on Geomagnetic Observatory Data Acquisition and Processing; Finnish Meteorological Institute: Helsinki, Finland, 1990. Available online: https:/ / www.gemsys.ca/pdf/Overhauser-Magnetometers-for-Measurement-of-the-Earth \T1 $\backslash$ textquoterightsMagnetic-Field.pdf (accessed on 27 October 2021).

15. Sapunov, V.; Denisov, A.; Denisova, O.; Saveliev, D. Proton and Overhauser magnetometers metrology. Contrib. Geophys. Geod. 2001, 31, 119-124.

16. Khomutov, S.Y.; Mandrikova, O.V.; Budilova, E.A.; Kusumita, A.; Manjula, L. Noise in raw data from magnetic observatories: DIMOVER. Earth Planets Space 2006, 58, 711-716. [CrossRef]

17. Jian, G.; Dong, H.; Liu, H.; Yuan, Z.; Dong, H.; Zhao, Z.; Liu, Y.; Zhu, J.; Zhang, H. Overhauser geomagnetic sensor based on the dynamic nuclear polarization effect for magnetic prospecting. Sensors 2016, 16, 806. [CrossRef]

18. Liu, H.; Bai, B.; Liu, Z. Contruction of an overhauser magnetic gradiometer and the applications in geomagnetic observation and ferromagnetic target localization. J. Instrum. 2017, 12, T10008. [CrossRef]

19. Liu, H.; Dong, H.; Liu, Z.; Ge, J.; Bai, B.; Zhang, C. Noise characterization for the FID signal from proton precession magnetometer. J. Instrum. 2017, 12, P07019. [CrossRef]

20. Liu, H.; Dong, H.; Liu, Z.; Ge, J. Application of Hilbert-Huang decomposition to reduce noise and characterize for NMR FID signal of proton precession magnetometer. Instrum. Exp. Tech. 2018, 61, 55-64. [CrossRef]

21. Bai, B.; Liu, H.; Ge, J.; Dong, H. Research on an improved resonant cavity for overhauser geomagnetic sensor. IEEE Sens. J. 2018, 18, 2713-2721. [CrossRef]

22. Liu, H.; Dong, H.; Ge, J.; Liu, Z.; Zhu, J.; Zhang, H. Efficient performance optimization for the magnetic data readout from a proton precession magnetometer with low-rank constraint. IEEE Trans. Magn. 2019, 55, 1-4. [CrossRef]

23. Acuna, M.H. Space-based magnetometers. Rev. Sci. Instrum. 2002, 73, 3717-3736. [CrossRef]

24. Lenz, J.; Edelstein, S. Magnetic sensors and their applications. IEEE Sens. J. 2006, 6, 631-649. [CrossRef]

25. Narkhov, E.D.; Sapunov, V.A.; Denisov, A.U.; Saveliev, D.V. Novel quantum NMR magnetometer non-contact defectoscopy and monitoring technique for the safe exploitation of gas pipelines. WIT Trans. Ecol. Environ. 2014, 186, 649-658.

26. Oluwagbemi, J.E.; Olakunle, A.S.; Lawrence, O.O. A study of subsurface geological structure of Sumaje village, Nigeria. J. Geol. Min. Res. 2013, 5, 232-238. [CrossRef] 
27. Hatakeyama, T.; Kitahara, Y.; Yokoyama, S.; Jigena, B.; Berrocoso, M.; Torrecillas, C.; Vidfal, J.; Barbero, I.; Fernandez-Ros, A. Magnetic survey of archaeological kiln sites with Overhauser magnetometer: A case study of buried Sue ware kilns in Japan. J. Archaeol. Sci. Rep. 2018, 18, 568-576. [CrossRef]

28. Gerginov, V.; Krzyzewski, S.; Knappe, S. Pulsed operation of a miniature scalar optically pumped magnetometer. J. Opt. Soc. Am. B Opt. Phys. 2017, 34, 1429-1434. [CrossRef]

29. Liwei, J.; Quan, W.; Liang, Y.; Liu, J.; Duan, L.; Fang, J. Effects of pump laser power density on the hybrid optically pumped comagnetometer for rotation sensing. Opt. Express 2019, 27, 27420-27430.

30. Savukov, I.; Kim, Y.J.; Shah, V.; Boshier, M.G. High-sensitivity operation of single-beam optically pumped magnetometer in a kHz frequency range. Meas. Sci. Technol. 2017, 28, 035104. [CrossRef]

31. Jodko-Wadzińska, A.; Wildner, K.; Pako, T.; Wladzinski, M. Compensation system for biomagnetic measurements with optically pumped magnetometers inside a magnetically shielded room. Sensors 2020, 20, 4563. [CrossRef]

32. Liu, L.; Lu, Y.; Zhuang, X.; Zhang, Q.; Fang, G. Noise analysis in pre-amplifier circuits associated to highly sensitive opticallypumped magnetometers for geomagnetic applications. Appl. Sci. 2020, 10, 7172. [CrossRef]

33. Volkmar, S.; Bastian, S.; Rob, I.; Scholtes, T.; Woetzel, S.; Stolz, R. An optically pumped magnetometer working in the light-shift dispersed mz mode. Sensors 2017, 17, 561. [CrossRef]

34. Gerginov, V.; Pomponio, M.; Knappe, S. Scalar magnetometry below $100 \mathrm{fT} / \mathrm{Hz} 1 / 2$ in a microfabricated cell. IEEE Sens. J. 2020, 20, 12684-12690. [CrossRef]

35. Jaufenthaler, A.; Kornack, T.; Lebedev, V.; Limes, M.E.; Korber, R.; Liebl, M.; Baumgarten, D. Pulsed optically pumped magnetometers: Addressing dead time and bandwidth for the unshielded magnetorelaxometry of magnetic nanoparticles. Sensors 2021, 21, 1212. [CrossRef] [PubMed]

36. Denisov, A.Y.; Denisova, O.V.; Sapunov, V.A.; Khomoutov, S.Y. Measurement quality estimation of proton-precession magnetometers. Earth Planets Space 2006, 58, 707-710. [CrossRef]

37. Guiberteau, T.; Grucker, D. Dynamic nuclear polarization of water protons by saturation of $\sigma$ and $\pi$ EPR transitions of nitroxides. J. Magn. Reson. Ser. A 1993, 105, 98-103. [CrossRef]

38. Breit, G.; Rabi, I.I. Measurement of nuclear spin. Phys. Rev. 1931, 38, 2082-2083. [CrossRef]

39. Lang, K.; Moussavi, M.; Belorizky, E. New, high-performance, hydrogenated paramagnetic solution for use in earth field DNP-NMR magnetometers. J. Phys. Chem. A 1997, 101, 1662-1671. [CrossRef]

40. Yamada, K.-I.; Kinoshita, Y.; Yamasaki, T.; Sadasue, H.; Mito, F.; Nagai, M.; Matsumoto, S.; Aso, M.; Suemune, H.; Sakai, K.; et al. Synthesis of nitroxyl radicals for overhauser-enhanced magnetic resonance imaging. Arch. Pharm. 2008, 341, 548-553. [CrossRef] [PubMed] 